\title{
Biomarcadores en espondiloartropatías
}

\author{
CONSUELO ROMERO-SÁNCHEZ ${ }^{1,2, a}$, JOHN LONDOÑO ${ }^{1}$, \\ JULIETTE DE AVILA ${ }^{2, b}$, RAFAEL VALLE-OÑATE ${ }^{1}$
}

\section{Biomarkers for spondyloarthropathies. State of the art}

\begin{abstract}
Among rheumatic diseases and specifically spondyloarthropathies (SpA), the study of biomarkers, defined as molecules that reflect either biologic or specific pathological process, is an important and necessary area in basic and clinical research, being a consequence or the response of an intervention. Other markers provide information about the pathogenesis of this disease. Recently, HLA-B27 has been used as diagnostic criteria to detect SpA. Bath Ankylosing Spondylitis Disease Activity Index (BASDAI) and Bath Ankylosing Spondylitis Functional Index (BASFI) are clinical scores used to assess disease activity. A new activity index, Ankylosing Spondylitis Disease Activity Score (ASDAS) considers erythrocyte sedimentation rate (ESR) and $C$-reactive protein $(C R P)$ as biomarkers. This review describes the state of the art of research on SpA biomarkers. There are promising new candidates as biomarkers such as metalloproteinase 3, Type II collagen neoepitopes (C2C and C1-2C), C-propeptide of Type II collagen (CPII), aggrecan 846 epitope, macrophage colony stimulating factor, serum amyloid A protein and interleukin-6, among others.
\end{abstract}

(Rev Med Chile 2010; 138: 1179-1185).

Key words: Biological markers; Joint diseases; Spondylitis.

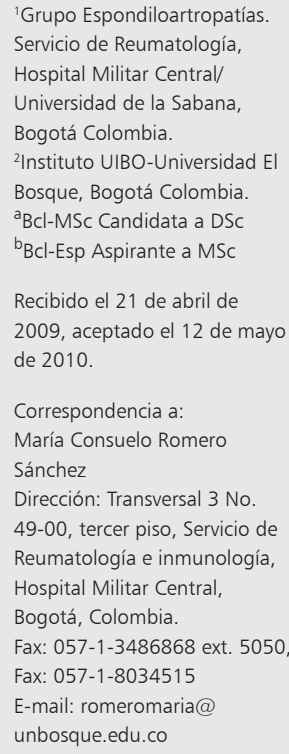

$\mathrm{E}$ 1 estudio de biomarcadores en las espondiloartropatías (EAS) se ha convertido en un tema de investigación importante dado que los utilizados comúnmente: la velocidad de sedimentación globular (VSG), la PCR y el HLAB27, tienen una baja sensibilidad y especificidad. La rápida progresión en los últimos años de los tratamientos con los denominados "agentes biológicos", demanda la disponibilidad en la práctica clínica de métodos que evidencien de una manera más segura los eventos relacionados con el diagnóstico, actividad, el tratamiento, la progresión y el pronóstico de la enfermedad. En la mayoría de los casos estos biomarcadores son utilizados en investigación, contribuyendo al mejor entendimiento de las EAS. En la actualidad existen candidatos promisorios a biomarcadores en estas enfermedades: la metaloproteinasa 3 (MMP-3), el neopéptido C2C del colágeno, el propéptido $\mathrm{C}$ de colágeno tipo II, el agrecan 846, el factor estimulante de colonias de macrófago, la proteína amiloide A del suero, la IL-17 y la IL 6, entre otras ${ }^{1-5}$.

Los biomarcadores son sustancias bioquímicas (aunque se puede considerar también el ARN, el $\mathrm{ADN}$, sus fragmentos o la combinación de ellos) que reflejan un proceso biológico o patológico específico, su consecuencia o la respuesta a una intervención. En EAS podemos pensar entonces en una estrategia similar a la propuesta en otras enfermedades reumáticas, donde se puede reconocer un momento biológico del paciente o de una intervención terapéutica y establecer la correlación con los valores de estos marcadores. Ellos pueden ser obtenidos de una variedad de componentes corporales como: sangre, orina, líquido sinovial, tejido sinovial, entre otros ${ }^{6-8}$.

El proceso de validación de un biomarcador depende de su capacidad para definir una enfermedad o un proceso relacionado con la misma. Basados en varias consideraciones se han clasifi- 
cado en los siguientes grupos: de diagnóstico, de severidad y/o de actividad de la enfermedad, de pronóstico, de eficacia de una intervención o de uso en investigación. En esta revisión se hará énfasis en los biomarcadores de utilidad diagnóstica, de seguimiento de la actividad y algunos de interés en investigación ${ }^{6}$.

Las EAS son un grupo heterogéneo de enfermedades articulares inflamatorias crónicas, que comparten manifestaciones clínicas, radiológicas, asociación con el HLA-B27 y una marcada tendencia a la asociación familiar. La presentación clínica de las EAS se caracteriza por compromiso del esqueleto axial, articular periférico, entesopatía y manifestaciones extra articulares. Recientemente se ha propuesto unificar el nombre de "Espondiloartritis" para enfatizar la naturaleza inflamatoria de estas patologías. El término es usado generalmente en plural ya que se refiere a un grupo de enfermedades similares con diferentes manifestaciones clínicas y una predisposición genética común, más que una sola enfermedad con diferentes presentaciones ${ }^{9}$.

Se han reconocido cinco entidades dentro del grupo de las EAS sobre la base de los criterios diagnósticos propuestos por el Grupo Europeo para el estudio de las espondiloartropatías: espondilitis anquilosante (EA), artritis psoríasica (APs), artritis reactiva ( $\mathrm{ARe})$, artritis asociada a enfermedad inflamatoria intestinal (IBD) y el grupo de espondiloartropatías indiferenciadas (EASI). Junto a la EA, las EASI son el subtipo más común con una prevalencia entre $0,7 \%$ y $2,0 \%$ en la población general $^{9}$. Sin embargo, en un estudio realizado por nuestro grupo demostró el incrementó en la presencia de ARe dado el contexto infeccioso en nuestro medio y la asociación descrita posterior a una infección por bacterias artritogénicas ${ }^{10}$.

Por medio de la metodología de consenso, se han agrupado las variables más representativas de los fenómenos inflamatorios relacionados con la actividad de la enfermedad, en conjuntos llamados dominios: función física, dolor, movilidad de la columna, evaluación global del paciente, rigidez, articulaciones, entesis, fatiga, radiografía de la columna y reactantes de fase aguda (PCR y VSG). Estos dominios pretenden medir el impacto de la enfermedad en los pacientes, así como su respuesta a diferentes modalidades terapéuticas, en ellos se utilizan instrumentos específicos de evaluación acuerdo a las características de las variables que los componen. Pueden emplearse en diversos escenarios clínicos o epidemiológicos como la efectividad de tratamientos sobre los síntomas y la progresión de la enfermedad, el almacenamiento de información o el efecto de programas integrales de rehabilitación. En el estudio de las EAS se utilizan los dominios de forma no restrictiva tal como ha sido recomendado por El Grupo ASAS (The Assessments in Ankylosing Spondylitis Working Group), durante la reunión IV de OMERACT (Outcome Meassures in Rheumatoid Artritis Clinical Trials) en 1998, donde esquematizó de manera sencilla cuales de los dominios serían indispensables para la valoración de cada escenario ${ }^{11}$.

\section{Marcadores diagnósticos}

Definidos como aquellas moléculas con capacidad de clasificar si un individuo en particular tiene o no la enfermedad. Si pensamos en EA, hasta hace un tiempo tendríamos que referirnos a los criterios de clasificación clínicos ${ }^{12}$, pero probablemente estos no sean adecuados como criterios diagnósticos por tener una baja sensibilidad y especificidad en pacientes con enfermedad temprana ${ }^{12,13}$.

Rudwaleit et al 2005, publicaron una aproximación de un algoritmo de diagnóstico temprano para determinar la probabilidad de que un individuo tenga EAS con compromiso axial. Para establecer este algoritmo se ponderaron diferentes parámetros clínicos, de laboratorio e imágenes. El único biomarcador considerado dentro de estos parámetros fue la molécula de HLA-B27 $7^{12}$.

Recientemente, el grupo ASAS, estableció nuevos criterios de clasificación para pacientes con espondiloartropatía axial, los cuales pueden mejorar la clasificación de los pacientes y pueden orientar a los reumatólogos en el diagnóstico de estas enfermedades. Como uno de los criterios mayores fue establecido el HLA-B27 el cual debe estar acompañado de dos o más de las siguientes características: dolor lumbar inflamatorio, artritis, entesitis, uveitis, dactilitis, psoriasis, enfermedad de Chron, adecuada respuesta a los AINES, antecedentes familiares de EAS, y valores elevados de PCR ultrasensible ${ }^{14}$.

\section{Biomarcadores de seguimiento de la actividad}

Los candidatos a biomarcadores con el propósito de medir la actividad de la enfermedad requieren de un patrón de oro. En el caso de las EAS el parámetro clínico comúnmente aceptado es 
el BASDAI. La PCR y la VSG presentan resultados muy variables y por lo tanto, una baja sensibilidad y especificidad ${ }^{11}$.

Con el fin de evaluar la actividad de la enfermedad, Sheehan y cols midieron la VSG, la PCR, la $\alpha$ lantitripsina, y $\alpha 2$ macroglobulina en 65 pacientes con EA, encontrando una correlación positiva entre ellas pero ninguna con la actividad clínica o el grado de limitación funcional de la columna vertebral $^{12}$, sin embargo, se halló una asociación positiva entre la PCR y la presencia de uveítis, sugiriendo que los fenómenos extra articulares son la causa más importante de su elevación en estos marcadores ${ }^{16}$.

Otro estudio que incluyó 20 pacientes con EA, analizó la correlación con la actividad inflamatoria de la enfermedad y los niveles séricos de: PCR, VSG, haptoglobina y $\alpha 2$ microglobulina. Se encontró únicamente una buena correlación entre PCR y el BASDAI $(r=0,556, p<0,05)$. De igual manera, los resultados obtenidos en otro estudio multicéntrico que incluyó 191 pacientes con EA, se comparó el valor de la VSG con el BASDAI, mostrando una sensibilidad de 63\% y un especificidad de 52\%. Para la PCR la sensibilidad y especificidad fue del $44 \%$ y $81 \%$ respectivamente, los valores predictivos positivos fueron bajos de 0,15 y $0,69^{17,18}$.

Un número cada vez mayor de candidatos potenciales se han descrito, sin embargo, la contribución individual parece ser pequeña en el contexto general. De esta manera, la probabilidad de combinación de múltiples biomarcadores parece una buena opción que necesita ser evaluada de manera estructurada como lo observamos en los siguientes resultados.

En un trabajo presentado por nuestro grupo y la División de Inmunología y Reumatología del Instituto de Chung Shan Medical University en Taiwan, en donde se analizaron 240 pacientes, no se encontró correlación entre la VSG y la PCR con los índices de actividad y función, BASDAI y BASFI ${ }^{19}$.

Otro candidato dentro del grupo de reactantes de fase aguda, que se ha considerado como marcador de actividad inflamatoria en las EAS, es la proteína amiloide A del suero (ASA). Esta proteína es un miembro de la familia de las apolipoproteínas, sintetizadas principalmente en el hígado y el tejido sinovial por monocitos y macrófagos activados ${ }^{20}$. Su relación con la actividad se evaluó en un estudio que incluyó 72 pacientes con EA junto a la VSG, la PCR los cuales se compararon con el BASDAI. Hubo una buena correlación entre los tres biomarcadores y la proteína amiloide A con el BASDAI, por lo que se propone como un candidato a biomarcador de actividad ${ }^{17}$.

Recientemente la MMP-3 ha sido considerado uno de los biomarcadores más promisorios. La degradación de los componentes de la matriz extracelular es una característica patológica de las artritis inflamatorias crónicas, así como es esencial para el desarrollo embrionario, morfogénesis, reproducción, reabsorción y remodelado tisular $^{21}$. En artritis las MMP son frecuentemente generadas por fibroblastos y macrófagos ${ }^{22}$, células sinoviales, neutrófilos y condrocitos ${ }^{23,24}$ en respuesta a citocinas pro inflamatorias como la IL-1 y el TNF-alfa. Las MMP han sido bien estudiadas en artritis reumatoide (AR), considerándose buenos biomarcadores de actividad. Recientemente, varios estudios han indicado que estas MMP particularmente la MMP-3 (estromielisina-1) puede ser utilizada como potencial candidato a biomarcador en EAS, tanto en actividad, como en respuesta al tratamiento ${ }^{3,25}$.

Varios estudios que han incluido pacientes con EA y que han analizado los valores séricos de MMP han encontrado diferencias significativas al clasificar los pacientes según su grado de actividad de enfermedad y compararlos con los niveles obtenidos en sujetos sanos ${ }^{26-28}$. Esta diferencia también se mantiene cuando se comparan los niveles de MMP obtenidos en muestras diferentes al suero como en tejido sinovial y líquido sinovial de algunos pacientes con EAS. Yang el al (2004) publica el uso de los niveles de MMP-3 como marcador de actividad en un grupo de 41 individuos con EA, encontrando una alta correlación con la VSG $(r=054 ; p=0,0001)$ y con el BASDAI $(r=0,48$, $\mathrm{p}=0,0007)^{3}$.

En el 2006 el grupo de Chen y colaboradores, reportó niveles incrementados de MMP-3 frente a sujetos sanos $(\mathrm{p}<0,001)$, además encontró diferencia entre los individuos con alta actividad de la enfermedad, al compararlos con los de bajo grado en el BASDAI ( $\mathrm{r}=0,366 ; \mathrm{p}=0,017$ ), con el BASFI $(r=0,344, p=0,026)$, con VSG $(\mathrm{r}=0,383 ; \mathrm{p}=0,012)$ y para PCR $(\mathrm{r}=0,416$; $\mathrm{p}=0,006)$. Se obtuvo tanto para la VSG y la PCR una sensibilidad de $61,5 \%$ y especificidad de $62,5 \%$ con un valor predictivo positivo (VPP) de 72,7\% 
y un valor predictivo negativo (PPN) de $50 \%$ y para la MMP-3 tanto la sensibilidad como la especificidad fueron mayores con $69,2 \%$ y de $68,8 \%$ respectivamente, con un VPP de $78,3 \%$ y VPN de 57,9 \%, el área bajo la curva frente al BASDAI (mayor o igual a 4) para la VSG fue de 0,74 , para la PCR de 0,631 y para la MMP-3 de 0,612 27 . Resultados similares se hallaron en el estudio de Yang en donde el área bajo la curva para MMP-3 fue de $0,75^{3}$, demostrándose así, que la MMP-3 puede proveer mejor sensibilidad y especificidad que los biomarcadores tradicionales para la medición de la actividad de la enfermedad (Tablas 2 y 3 ).

La literatura nos muestra una vez más que a

Tabla 1. Biomarcadores diagnósticos en espondiloartropatías

1 HLA B 27 *

*Acompañado de 2 o más criterios clínicos establecidos por el grupo ASAS.

Tabla 2. Biomarcadores aceptados en actividad en espondiloartropatías

1 Velocidad de sedimentación globular

2 Proteínas $\mathrm{C}$ reactiva

Tabla 3. Biomarcadores propuestos en actividad en espondiloartropatías

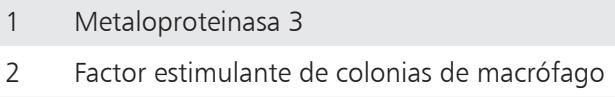

Tabla 4. Biomarcadores propuestos en investigación en espondiloartropatías

\begin{tabular}{|ll}
\hline 1 & Citocinas perfil Th 17( IL-17,IL-23, IL-6) \\
\hline 2 & Proteínas de unión al lipopolisacarido LBP \\
\hline 3 & Receptor CD 14 \\
\hline 4 & Neopéptido C2C del colágeno \\
\hline 5 & Propéptido C de colágeno tipo II \\
\hline 6 & Agrecan 846 \\
\hline 7 & IL-7 \\
\hline
\end{tabular}

pesar de buscar nuevas alternativas serológicas que reflejen fácilmente la actividad de las EAS, aún no se ha logrado reemplazar la PCR y la VSG. En la actualidad el grupo ASAS propuso un nuevo índice para la evaluación de actividad de la EA denominado ASDAS, considerado confiable y con capacidad de discriminar; incluye dominios y parámetros que son considerados de relevancia tanto para los pacientes como para el médico. Fueron establecidos los siguientes dominios: dolor, inflamación, pruebas de laboratorio, evaluación global del paciente, signos periféricos y fatiga; dentro del dominio de pruebas de laboratorio, continúan siendo considerados la PCR ultrasensible y VSG como biomarcadores que reflejan el estado de actividad del paciente ${ }^{28}$.

\section{Biomarcadores de investigación}

Un biomarcador en investigación es considerado cuando no hay información suficiente para permitir su inclusión en una de las categorías existentes. Esto puede ser representado por un genotipo, un ensayo de una molécula o fragmento liberado, por ejemplo dentro del líquido sinovial, o en circulación en donde su relación aún no ha sido establecida. El propósito de crear esta clasificación es facilitar y encontrar la codificación de potenciales biomarcadores, y por ende generar aún más el desarrollo de la investigación encaminada a tratar de establecer un papel para estos en una o varias de las categorías mencionadas ${ }^{6}$ (Tabla 4 ).

En la investigación para la búsqueda de biomarcadores, conociendo la naturaleza de la inflamación en estas patologías, se debe considerar la producción de citocinas en donde existe resultados preliminares por nuestro grupo ${ }^{30,31}$. En esta línea los resultados han sido variables y difíciles de interpretar clínicamente.

Dentro de las citocinas propuestas como biomarcadores esta principalmente la IL-6 la cual es una citocina pleiotrópica, reconocida por la inducción de la síntesis de una variedad de proteínas hepáticas, varias veces mencionadas como reactantes de fase aguda, implicados como marcadores biológicos como la PCR, proteína amiloide A del suero, fibrinógeno, complemento, alfa1-antitripsina, etc ${ }^{32}$. Los niveles de IL-6 se han encontrado incrementados en pacientes con EA comparados con sujetos sanos, encontrándose correlación de la IL-6 con la anquilosis vertebral ${ }^{33,34}$ aún más en pacientes con actividad de la enfermedad. Sus 
niveles se han correlacionado con VSG $(r=0,57$, $\mathrm{p}<0,0011)$, PCR $(\mathrm{r}=0,005, \mathrm{p}>0,0011)$, número de plaquetas $(\mathrm{r}=0,43, \mathrm{p}>0,0011)$ y el índice de rigidez análoga visual (VAS) $(r=0,37, p=0,022)^{30}$. En otra cohorte de 70 pacientes se demostró una correlación adecuada entre los niveles de IL-6 y VSG $(r=0,385, p=0,001)$ y con la PCR $(r=0,253$, $\mathrm{p}=0,034)^{19,30}$. En un estudio previo realizado por nuestro grupo se compararon niveles de citocinas en líquido sinovial y suero de 19 pacientes con EASI encontrándose únicamente niveles altos en el fluido estadísticamente significativos para IL-6, IL-1 alfa e IP10 (citocina quimioatractante para monocitos/macrófagos, linfocitos T, NK y células dendríticas), estos resultados inducen a generar propuestas en donde el bloqueo de la IL-6 puedan tener consecuencias interesantes en los pacientes con $\mathrm{EAS}^{31}$.

Usando La tecnología de microarreglos la cual consiste en el análisis de múltiples genes de manera diferencial y ordenada, Yang y col investigaron 1.176 genes en tejido sinovial de pacientes con EAS encontrando la presencia incrementada de otro biomarcador interesante, el factor estimulante de colonias de monocito M-CSF, además de la MMP-3 y la IL-7. De estos biomarcadores las concentraciones séricas de MMP-3 y de M-CSF correlacionaran con actividad de la enfermedad. Los autores hacen énfasis en la importancia del hallazgo dado que resultados de esta citocina no habían sido reportados en pacientes con $\mathrm{EAS}^{3}$.

Recientemente como biomarcador en investigación vale destacar a la IL-17, desde hace cinco años surge gran cantidad de información sobre la subpoblación de linfocitos T CD4 Th-17, sus citocinas relacionadas, receptores y sus efectos ${ }^{35}$. En el 2006 tres estudios encontraron que la combinación de TGF- $\beta$ y la citocina pleiotrópica IL-6, es necesaria para inducir la producción de IL-17 sobre células T nativas ${ }^{36-38}$. Además, las combinaciones de IL-6 o TGF- $\beta$ más IL-21, de IL- $1 \beta$ más IL-6, o de IL-1 $\beta$ más IL-23, se han propuesto como factores inductores de Th-17 en humanos.

Estudios recientes demuestran que el modelo clásico de las células T CD4 efectoras Th-1/Th-2 debe ser reinterpretado, dándole espacio a las células Th-17 dentro de las patogenias inflamatorias articulares como las EAS. Hasta el momento la evidencia permite considerar a los linfocitos Th-17 como células altamente diferenciadas, que expresan moléculas de superficie y receptores in- volucrados en su migración hacia el tejido sinovial generando reacciones inmunes autodestructivas locales especialmente en articulación, permitiendo el desarrollo de artritis. Una vez en el sitio de la inflamación los sinoviocitos pueden reclutar más linfocitos Th-17 a través de la producción de la quimiocinas como la CCL-20, con lo cual se incrementa la producción de citocinas pro inflamatorias como IL-17, IL-1, IL-6, TNF- $\alpha$, MMP, entre otras. Algunos productos relacionados con las células Th-17 tienen importantes y diversos efectos sobre células tales como monocitos/macrófagos, células dendríticas, células endoteliales, osteoblastos, fibroblastos, condrocitos entre otras. Uno de los principales efectos biológicos de estas citocinas es el reclutamiento de diversos tipos celulares en sitios de inflamación ${ }^{39-43}$.

Finalmente existe un tema de interés y son los resultados de los biomarcadores que reflejan el recambio de cartílago, aunque estos marcadores son más utilizados en osteoartrosis, el cartílago también es un órgano blanco importante en las $\mathrm{EAS}^{44}$. Los biomarcadores de la síntesis y degradación de cartílago son índices potenciales que reflejen actividad de la enfermedad. La degradación del colágeno tipo II, que es el principal componente del cartílago articular, genera nuevos epítopes como son las moléculas denominada $\mathrm{C} 2 \mathrm{C}$ y $\mathrm{C} 1-\mathrm{sC}^{45,46}$ son comúnmente liberados posterior al proceso de degradación. Otras moléculas que son reflejo de la síntesis del colágeno es el propéptido tipo II del procolágeno (CPII) y el agrecan considerado el principal componente de los proteoglicanos del cartílago, todas estas proteínas fueron medidas en el suero de pacientes con EA y se encontró que los niveles de ellas estaban significativamente elevadas con respecto a los controles ${ }^{47} \mathrm{y}$ al establecer un índice entre el CPII:C2C se encontró correlación con la PCR.

\section{Conclusión}

Existen cada día más diversidad de vías y moléculas que pueden adicionarse en el conocimiento de la patogenia y el monitoreo de la actividad inflamatoria de las EAS. Poder establecer el diagnóstico y el seguimiento de la actividad, nos permite implantar conductas de tratamiento adecuadas y oportunas, además de evidenciar la respuesta del paciente frente al tratamiento y progresión de la 
enfermedad disminuyendo recursos. Un número cada vez mayor de candidatos potenciales ha ido apareciendo, sin embargo, la contribución estadística individual parece ser pequeña en el contexto general. De esta manera, la probabilidad de combinación de múltiples biomarcadores es requerida.

\section{Referencias}

1. Lange U, Boss B, Teichmann J, Klor HU, Neeck G. Serum amyloid A-an indicator of inflammation in ankylosing spondylitis. Rheumatol Int 2000; 19: 119-22.

2. Sukenik S, Henki J, Zumlichman S, et al. Serum and synovial fluid levels of serum amyloid A protein and C reactive protein in inflammatory and non inflammatory arthritis. J Rheumatol 1998;15: 942-5.

3. Yang C, Gu J, Rihl M, Baeten D, Huang F, Zhao M, et al. Serum levels of matrix Metalloproteinase 3 and Macrophage colony stimulating factor 1 corrrelate with disease activity in ankylosing spondylitis. Arth Care Res 2004; 51: 691-9.

4. Visvanathan S, Wagner CL, Marini JC, Van der Heijde D, Baker D, Gathany J, et al. Inflammatory biomarkers, disease activity, and spinal disease measures in patients with ankylosing spondylitis after treatment with infliximab. Ann Rheum Dis 2008; 67: 511-7.

5. Manicourt DH, Fujimoto N, Obata K, Thonar EJ. Levels of circulating collagenase, stromelysin-1, and tissue inhibitor of matrix metalloproteinases 1 in patients with rheumatoid arthritis. Relationship to serum levels of antigenic keratan sulfate and systemic parameters of inflammation. Arthritis Rheum 1995; 38: 1031-9.

6. Bauer DC, Hunter DJ, Abramson SB, Attur M, Corr M, Felson D, et al. Osteoarthritis Biomarkers Network. Classification of Osteoarthritis biomarkers proposed approach. Osteoarthritis Cartilage 2006; 14: 723-7.

7. Huang F, Zhu J, Zhang L, Zhang J, Zhang F, Yu D. Response to one infusion predicts subsequent improvement as well as the rate of relapse of ankylosing spondylitis infused with three pulses of infliximab. Clin Rheumatol 2007; 26: 920-6.

8. Kruithof E, De Rycke L, Vandooren B, De Keyser F, Fitz Gerald O, McInnes I, et al. Identification of synovial biomarkers of response to experimental treatment in early-phase clinical trials in spondylarthritis. Arthritis Rheum 2006; 54: 1795-804.

9. Zochling J, van der Heijde D, Burgos-Vargas R, Collantes E, Davis JC, Dijkmans B, et al. ASAS/EULAR recommendations for the management of ankylosing spondylitis. Ann Rheum Dis 2006; 65: 442-52.

10. Valle R, Londoño J, Avila M, Iglesias A, Cuellar ML. Es- pinosa L. Outcome of patients with undifferentiated seronegative spondyloarthropathy. Arthritis Rheum1997; 40 (supl. 1): 270.

11. Van der Heijde D, Calin A, Dougados M, Khan M, van der Linden S, Bellamy N. Which domains should be included in a core set for endpoints in Ankkylosing Spondylitis? Introduction to the Ankylosing Spondylitis module of OMERACT IV. J Rheumatol 1999; 26: 945-50.

12. Zochling J, Brand J and Braun J. The current concept of spondyloarthritis with special emphasis on undifferentiated spondyloarthritis Rheumatol 2005; 44: 1483-91.

13. Rudwaleit M, Khan MA, Sieper J. The challenge of diagnosis and classification in early ankylosing spondylitis: do we need new criteria? Arthritis Rheum 2005; 52: 1000-8.

14. Rudwaleit M, Van der Heijde D, Landewé R, Listing J, Akkoc N, Brandt J, et al. The Development of Assesment Spondyloarthritis International Society (ASAS) classification criteria for axial spondyloarthritis (part II):Validation and final selection. Ann Rheum Dis 2009; 68: 777-83.

15. Sheehan NJ, Slavin BM, Donovan MP, Mount JN, Mathews A. Lack of correlation between clinical disease activity and erythrocyte sedimentation rate, acute phase proteins or protease inhibitors in ankylosing spondylitis. Br J Rheumatol 1986; 25: 171-4.

16. Laurent MR, Panayi GS. Acute phase and serum immunoglobulins in ankilosing spondylitis. Ann Rheum Dis 1983; 42: 524-8.

17. Lange U, Boss B, Teichmann J, Klor HU, Neeck G. Serum amyloid A-an indicator of inflammation in ankylosing spondylitis. Rheumatol Int 2000; 19: 119-22.

18. Dougados M, Gueguen A, Nakache JP, Velicitat P, Zeidler $\mathrm{H}$, Veys $\mathrm{E}$, et al. Clinical relevance of C-reactive protein in axial involvement of ankylosing spondylitis. J Rheumatol 1999; 26: 971-4.

19. Wei JC, Avila M, Romero MC, Londoño J, Valle R, Yu D. Biomarkers for ankylosing Spondylitis. Revista Colombiana de Reumatología 2007; 14: 48.

20. Uhlar CM, Whitehead AS. Serum amyloid A, the major vertebrate acute-phase reactant. Eur J Biochem 1999; 265: 501-23.

21. Nagase H, Woessner JF. Matrix Metalloproteinases. J Biol Chem 1999; 274: 214914.

22. Grillet B, Dequeker J, Paemen L, Van Damme B and Opdenakkeer G. Gelatinase B in chronic synovitis: immunolocalization with a monoclonal antibody. $\mathrm{Br} \mathrm{J}$ Rheumatol 1997; 36: 744-47

23. Cole AA, Chubinskaya S, Schumacher B, Huch K, Cs-Szabo G, Yao Jianling, et al. Chondrocyte matrix metalloproteinase-8. Human articular chondrocytes express 
neutrophil collagenase. J Biol Chem 1996; 271: 11023-6.

24. Mohtai M, Smith RL, Schurman DJ, Tsuji Y, Torti F, Hutchinson NI, et al. Expression of 92-kD type IV collagenase/gelatinase (gelatinase B) in osteoarthritic cartilage and its induction in normal human articular cartilage by interleukin 1. J Clin Invest 1993; 92: 179-85.

25. Vandooren B, Kruithof E, Yu DT, Rihl M, Gu J, De Rycke $\mathrm{L}$, et al. Involvement of matrix metalloproteinases and their inhibitors in peripheral synovitis and downregulation by tumor necrosis factor alpha blockade in spondylarthropathy. Arthritis Rheum 2004; 50: 2942-53.

26. Chen $\mathrm{CH}$, Liao HT, Chen HA, Liang TH, Wang HP, Chou CT. Serum levels of matrix metalloproteinase- 3 in undifferentiated spondyloarthropathy. Scand J Rheumatol 2007; 36: 326-8.

27. Chen CH, Lin KC, Yu DTY, Yang C, Huang F, Cjen HA, et al. Serum matrix metalloproteinases and tissue inhibitors of metalloproteinases in ankylosing spondylitis: MMP3 is a reproducible sensitive and specific biomarker of disease activity. Rheumatol 2006; 45: 414-20.

28. Lukas C, Landewé C, Sieper J, Dougados M, Davis J, Braun J. Development of an ASAS-endorsed disease activity score (ASDAS) in patients with ankylosing. Ann Rheum Dis 2009; 68: 18-24.

29. Uhlar CM, Whitehead AS. Serum amyloid A, the major vertebrate acute-phase reactant. Eur J Biochem 1999; 265: 501-23.

30. Bal A, Unlu E, Bahar G, Aydog G, Eksioglu E, Yorgansioglu. Comparison of serum IL-1 beta, sIL-2R, IL-6, and TNF-alpha levels with disease activity parameters in ankylosing spondylitis. Clin Rheumatol 2007; 26: 211-5.

31. Romero-Sánchez C, Robinson W, Tomooka B, Londoño J, Valle-Oñate R, Huang Feng, et al. identification of Acute phases reactants and cytokines useful for monitory infliximab therapy in ankylosyng spondylitis. Clin Rheumatol 2008; 27: 1429-35.

32. Ershler WB, Keller ET. Age associated increased IL-6 gene expression, late life disease, and frailty. Ann Rev Med 2000; 52: 245-79.

33. Falkenbach A, Herold M. In ankylosing spondylitis serum interleukin- 6 correlates with the degree of mobility restriction, but not with short-term changes in the variables for mobility. Rheumatol Int 1998; 18: 103-6.

34. Gratacós J, Collado A, Filella X, Sanmarti R, Cañete J, LLena J, et al. Serum cytokines (IL-6, TNF-alpha, IL-1 beta and IFN-gamma) in ankylosing spondylitis: a close correlation between serum IL- 6 and disease activity and severity. Br J Rheumatol 1994; 33: 927-31.
35. Korn T, Bettelli E, Oukka M, Kuchroo VK. IL-17 and Th17 Cells. Annu Rev Immunol 2009; 27: 485-517.

36. Veldhoen M, Hocking RJ, Atkins CJ, Locksley RM, Stockinger B. TGFbeta in the context of an inflammatory cytokine milieu supports de novo Differentiation of IL-17-Producing T Cells. Immunity 2006; 24: 179-89.

37. Wynn TA. $\mathrm{T}(\mathrm{H})-17$ : a giant step from $\mathrm{T}(\mathrm{H}) 1$ and $\mathrm{T}(\mathrm{H}) 2$. Nat Immunol 2005; 6: 1069-70.

38. Mangan PR, Harrington LE, O'Quinn DB, Helms WS, Bullard DC, Elson CO, et al. Transforming growth factor-beta induces development of the TH17 lineage. Nature 2006; 441: 231-4.

39. Layh-Schmitt G, Colbert RA. The interleukin-23/interleukin-17 axis in spondyloarthritis. Curr Opin Rheumatol 2008; 20: 392-7.

40. Hirota K, Yoshitomi H, Hashimoto M, Maeda S, Teradaira S, Sugimoto N, et al. Preferential recruitment of CCR6-expressing Th17 cells to inflamed joints via CCL20 in rheumatoid arthritis and its animal model. J Exp Med 2007; 204: 2803-12.

41. Jandus C, Bioley G, Rivals JP, Dudler J, Speiser D, Romero P. Increased numbers of circulating polyfunctional Th17 memory cells in patients with seronegative spondylarthritides. Arthritis Rheum 2008; 58: 2307-17.

42. Shen H, Goodall JC, Hill Gaston JS. Frequency and phenotype of peripheral blood Th17 cells in ankylosing spondylitis and rheumatoid arthritis. Arthritis Rheum 2009; 60: 1647-56.

43. DeLay ML, Turner MJ, Klenk EI, Smith JA, Sowders DP, Colbert RA. HLA-B27 misfolding and the unfolded protein response augment interleukin-23 production and are associated with Th17 activation in transgenic rats. Arthritis Rheum 2009; 60: 2633-43.

44. Rousseau JC, Delmas PD. Biological markers in osteoarthritis. Nat Clin Pract Rheumatol 2007; 3: 346-56.

45. Billinghurst RC, Dahlberg L, Ionescu M, Reiner A, Bourne R, Rorabeck C, et al. Enhanced cleavage of type II collagen by collagenases in osteoarthritic articular cartilage. J Clin Invest 1997; 99: 1534-45.

46. Kojima T, Mwale F, Yasuda T, Girad C, Poole AR, Laverty $S$, et al. Early degradation of type IX and type II collagen with the onset of experimental inflammatory arthritis. Arthritis Rheum 2001; 44: 120-7.

47. Kim TH, Stone M, Payne U, Zhang X, Ionescu M, Lobanok T, et al. Cartilage biomarkers in ankylosing spondylitis: relationship to clinical variables and treatment response. Arthritis Rheum 2005; 52: 885-91. 